\title{
Consecuencias de la crisis financiera y social sobre la gobernanza del Modelo Social Europeo y el papel de la evaluación
}

\author{
Francisco González- Blanch \\ Universidad Complutense de Madrid \\ Francisco.gonzalez-blanch@ec.europa.eu
}

\begin{abstract}
Resumen
La gobernanza económica es una prioridad en las políticas comunitarias especialmente hoy, ante una crisis que confiere una imprevisibilidad excepcional en el porvenir de la economía europea. El examen de las causas de la crisis ha destacado diversas debilidades del Modelo Social Europeo. Los instrumentos de gobernanza del MSE (directivas, Directrices Integradas, Método Abierto de Coordinación, Diálogo social, aprendizaje mutuo, programas comunitarios) deben revisarse para que sean pertinentes en la situación actual. La capacidad de la Unión Europea y los Estados miembros para prever, anticipar y adaptar las competencias requeridas a las necesidades del mercado de trabajo constituye una condición esencial para la elaboración de políticas de empleo eficaces. La evaluación puede aportar criterios e instrumentos útiles para desarrollar la capacidad de gobernanza de la política de empleo con el fin de hacer frente a las necesidades actuales del mercado de trabajo y posibles reformas.
\end{abstract}

Palabras clave Gobernanza, Directrices Integradas, Estrategia de Lisboa Renovada, Pacto de Estabilidad y Crecimiento, empleabilidad, flexiseguridad, Modelos de Bienestar Social, Fondo Social Europeo, Evaluación de Impacto, Enfoque Asociativo, Método Abierto de Coordinación (OCM), Modelo Social Europeo, Plan de Recuperación Europa 2020.

\section{EU Governance capacity for responding to economic crisis through evaluation and prevention of future labor market needs}

\footnotetext{
Abstract

Economic governance has become a priority in Community policies and more today, before the crisis, which confers a unique unpredictability of the future of the European economy. Examination of the crisis has highlighted several weaknesses in the governance of the European Social Model. The various policy instruments of governance in ESM (directives, Integrated Guidelines, OMC, social dialogue, mutual learning and community programs) should be reviewed to make them relevant in the current situation. The capacity of the EU and its MMSS to foresee, anticipate and match the required skills and the needs of the labour market is a sine qua non in developing effective policies in employment, education and training and the optimal personal career choices. The evaluation can provide useful tools for developing of EU governance capacity. In this article we examine the existing capacities within the EU to achieve an appropriate strategy for cooperation between MS in order to meet current needs of labour market.

\section{Key words}

The Stability Governance Integrated Guidelines, the renewed Lisbon Strategy and Growth Pact, Employability, Flexicurity, Models of Social Welfare, the European Social Fund, Impact Assessment, Partnership Approach, OMC (CMO), the European Social Model Plan Recovery Europe 2020.
} 


\section{PRINCIPALES INSTRUMENTOS DE GOBERNANZA EN LA ESTRATEGIA DE LISBOA RENOVADA}

- Directrices Integradas de política económica y empleo adoptadas por el Consejo en el 2005 y actualizadas en el 2008, son 24 Directrices diseñadas como instrumento de coordinación y orientación política.

- Programas Nacionales de Reforma de Documentos preparados por los Estados miembros por periodo de tres años. Cada año se actualizan a través de informes de realización.

- Recomendaciones especificas para un Estado miembro (EM). Lo realiza el Consejo sobre la base de recomendaciones de la Comisión.

- Informe de Progreso Anual de la Comisión Evaluación anual de la Comisión sobre los progresos hechos sobre el cumplimiento de la estrategia.

- Método Abierto de Coordinación. Método intergubernamental "Coordinación suave" por la cuál un Estado miembro es evaluado por otro y la Comisión juega el papel de vigilancia.

\section{LA CRISIS ECONÓMICA Y LA EVALUACIÓN DE LA ESTRATEGIA DE LISBOA RENOVADA}

La crisis económica, sin precedentes, que desde otoño de 2008, golpea a la economía real europea, está suponiendo desafíos enormes para la Unión Europea (UE). Esta crisis está afectando a los puestos de trabajo, a las empresas y al comercio, dando lugar a una disminución de la actividad económica y a un declive en el crecimiento económico con el correspondiente aumento del desempleo. El impacto de la crisis está teniendo efectos profundos y duraderos en las economías de Europa. En la UE el PIB disminuyó un $4 \%$ en el 2009, se espera que la tasa de paro alcance el 8,6\% en 2009 y está previsto que continúe aumentando en el 2010 (9,5\%), con un desempleo juvenil superior al $21 \%$.

Para combatir la crisis, la UE está aplicando el "Plan de recuperación económica para Europa”, aprobado por el Consejo Europeo en diciembre de 2008. Incluye incentivos fiscales del orden al menos de 200.000 millones de euros (el 1,5 del PIB de la UE) y un plan de acción a corto plazo centrado en tres puntos:

- Una nueva arquitectura de los mercados financieros a nivel de la UE (informe Larosière).

- Medidas para responder a los efectos de la economía real.

- Una respuesta global a la crisis financiera. 
Y todo ello, definiendo la solidaridad y la justicia social como principio rector fundamental, que en el ámbito laboral se traduce en que hay que adoptar las medidas necesarias para proteger el empleo y, por otro lado mejorar las perspectivas laborales de las personas que lo pierden.

La crisis ha reducido el potencial de crecimiento de la UE a la mitad, tal vez puede que termine la década con un crecimiento económico muy bajo. Los niveles de productividad se están quedando rezagados: dos tercios de la diferencia de renta de la UE con respecto a Estados Unidos se debe a la menor productividad.

La UE está afrontando las diferentes etapas de la crisis de manera unida coordinando sus esfuerzos para transformar los desafíos en oportunidades. Los Estados miembros y la Comisión, en estrecha colaboración, han acordado una serie de medidas inmediatas que deben aplicarse de modo puntual, selectivo y temporal, respetando los siguientes principios rectores: fomentar la apertura, dentro del mercado interior y con respecto a terceros países; evitar la discriminación de productos y servicios de otros Estados miembros; mantener la coherencia con los objetivos de reforma a largo plazo. La actual prioridad es seguir apoyando la demanda y frenar el crecimiento del desempleo, lo que significa aplicar con energía el Programa Europeo de Recuperación Económica. Es esencial utilizar todos los instrumentos posibles para frenar la pérdida de empleo y ayudar a todos los que se encuentran sin trabajo.

A pesar de que el grueso de las competencias de la política de empleo depende de los Estados miembros, a la Comisión le corresponde desempeñar un papel importante, tiene que asegurar que las propuestas frente a la crisis alcancen realmente sus objetivos y poder revisarlas y corregirlas cuando no funcionen con arreglo a las expectativas. Se produce, por tanto, un intento por parte de la Comisión de conciliar los planteamientos generales del mercado único europeo y los enfoques nacionales para hacer frente a la crisis, sobretodo en la lucha por combatir el paro.

En la crisis actual, la Estrategia de Lisboa renovada en el 2005 y centrada en el crecimiento y el empleo, está siendo objeto de evaluación. Dicha estrategia estableció una nueva estructura de gobernanza basada en un enfoque de asociación entre los Estados miembros y las instituciones de la UE e incluía unas directrices integradas de política económica y social, que siguen siendo el marco efectivo para favorecer el crecimiento y el empleo de modo sostenible.

La Estrategia de Lisboa ha tenido un impacto positivo en la UE, aunque no se hayan alcanzado sus objetivos principales (es decir, un nivel de empleo del $70 \%$ y un gasto del I+D del 3\% del PIB). El nivel de empleo de la UE alcanzó el 66\% en 2008 (frente al $62 \%$ de 2000 ), antes de volver a caer a consecuencia de la crisis.

La renovación de la estrategia contribuyó a aclarar los objetivos de la UE. En particular, la definición de cuatro áreas prioritarias (investigación e innovación; in- 
versión en las personas y modernización de los mercados de trabajo; apertura del potencial empresarial, particularmente de las PYMES; energía y cambio climático). La Estrategia de Lisboa renovada no debía quedar relegada a un segundo plano por la situación coyuntural, bien es cierto que debería haber sido organizada mejor, centrándose en aquellos elementos que han jugado un papel clave en la crisis, tales como la supervisión de los mercados financieros, burbujas especulativas (mercado inmobiliario), créditos al consumo, aumentos salariales por encima de la productividad, dando lugar a elevados déficit por cuenta corriente. Ahora en el 2010, hay que revisarla, para ajustarla a las prioridades del 2020 y transformarla en una estrategia que plasme la visión integrada para la Europa de los 27. Es decir, una estrategia de convergencia y coordinación que permita alcanzar una visión integrada de la UE. En las economías del área euro interconectadas, sólo se aprovechará el potencial de crecimiento y empleo si todos los Estados miembros aplican las reformas más o menos al mismo ritmo.

La crisis económica ha atraído la atención a esta interdependencia en una economía estrechamente integrada, particularmente en la eurozona y ha evidenciado la necesidad de proseguir y acelerar las reformas estructurales. El impacto muy diverso de la crisis en la eurozona ha puesto de manifiesto que algunos países han progresado mucho más que otros en cuanto a la aplicación de sus programas de reformas estructurales y al mantenimiento de su competitividad.

En el ámbito del empleo, se constata que persisten problemas estructurales en muchos Estados miembros, como el hecho de que en general los mercados de trabajo europeos están segmentados, el desempleo juvenil es comparativamente elevado en relación a otras economías y la participación en el aprendizaje permanente es todavía pequeña. A la Comisión le corresponde el papel de impulsar la modernización significativa de las cualificaciones y la calidad de la formación, haciendo un inventario de las cualificaciones para el futuro, utilizando los programas de la UE para ayudar a los Estados miembros, y jugando la baza del presupuesto de la UE para adaptar las normas de financiación de la UE para enfrentarse al desempleo.

La crisis está también poniendo a prueba otros instrumentos de la UE, vinculados a la estrategia de Lisboa como el Pacto de Estabilidad y Crecimiento (PEC), aprobado por el Consejo Europeo en junio del 2004, y que constituye un pilar fundamental sobre el que se apoyan los instrumentos de la gobernanza económica de la UE. EI mantenimiento de unas finanzas públicas saneadas es una obligación del Tratado, por parte de los Estados miembros y se refleja con una coordinación de los políticas fiscales a través del Pacto de Estabilidad. Sin embargo, la crisis económica ha alterado radicalmente todo esto, con un dramático impacto sobre las finanzas públicas de los Estados miembros.

El déficit presupuestario que alcanzo el 2\% del PIB en el 2008 en la zona Euro, y se prevé que llegue a una media del $7 \%$ en el 2009 y la deuda se aproximará al $80 \%$ 82 del PIB, un incremento de alrededor de 20 puntos porcentuales en 2 años. Esto in- 
dica que la consolidación presupuestaria propuesta en el Pacto ha sido insuficiente. El Consejo Europeo ha pedido a los Estados miembros que, a pesar de la crisis, deben de volver, lo antes posible a sus objetivos presupuestarios a medio plazo de conformidad con el Pacto de Estabilidad y Crecimiento, recuperando así lo antes posible posiciones consecuentes con la sostenibilidad de la hacienda publica europea. Ello va a requerir una vigilancia presupuestaria más amplia y profunda por parte de la Comisión, y una mayor profundización sobre la coordinación de las políticas económicas.

Aunque la estabilidad macro financiera es un elemento fundamental para que tenga éxito la estrategia de salida de la crisis, el rápido aumento del desempleo es causa de grave preocupación. Es esencial utilizar todos los instrumentos posibles en la UE para frenar la pérdida de empleo y ayudar a los que se encuentran sin trabajo. A este respecto, la Comisión Europea señaló en el año 2009 las dos prioridades de actuación en materia de política de empleo que requerían una acción inmediata:

- Mejorar la empleabilidad a través de la flexiseguridad (combinación de flexibilidad y seguridad). La flexiseguridad ha sido uno de los más importantes logros políticos de la Estrategia de Lisboa renovada. Lanzada en el Consejo Europeo del 2007, es un concepto que reconoce que la globalización y el progreso tecnológico están rápidamente cambiando las necesidades de trabajadores y empresas. La flexiseguridad es definida como una estrategia integrada para promover la creación de mercados laborales más abiertos, más receptivos y más inclusivos que superen los problemas de la segmentación. Es un concepto que afecta tanto a quienes trabajan como a quienes están sin empleo o al margen del mercado, en especial de las personas más vulnerables. Más que proteger un trabajo, la flexiseguridad parte de la presunción de que es el trabajador quien necesita protección y asistencia para moverse hacia nuevos trabajos o continuar de manera actualizada en el mismo. Los principios comunes de la flexiseguridad se basan en el ofrecimiento de mejores oportunidades, incentivos económicos y medidas de apoyo que faciliten el acceso al empleo o avanzar hacia puestos de trabajo estables y con los elementos de seguridad previstos por la ley.

- La segunda prioridad consiste en mejorar los niveles de capacidades y su adecuación a las necesidades del mercado laboral. Para ello, se propone impulsar estrategias y medidas de activación para prevenir y limitar la pérdida de puestos de trabajo y sus efectos sociales negativos, garantizando el apoyo de unos sistemas de protección social para los que han perdido el empleo. Estos sistemas son fundamentales ya que desempeñan plenamente una función de estabilizadores automáticos para restablecer la confianza y ayudan a preparar el camino de la recuperación.

Pero el problema está en cómo articular estas dos prioridades, porque no es fácil de encajarlas en contextos económicos y políticos diferentes. Diversos estudios de 
evaluación sobre este debate (Comisión Europea, 2007) han llegado a reconocer que no existe contradicción en principio entre las reformas del mercado laboral que buscan fortalecer la flexibilidad y el dinamismo del mercado de trabajo con el objetivo de mejorar los niveles de capacidad de los empleos.

Vinculada a estas prioridades, la Comisión apoya, ante la situación de crisis, la movilidad laboral, que ha demostrado ser un importante factor de crecimiento económico. La movilidad incrementa las oportunidades de los trabajadores de encontrar empleo, pero sigue estando bastante restringida dentro de la Unión Europea. La política de emigración (de la UE y de los diferentes países) juega un papel importante para establecer la estructura que permitirá registrar diferentes formas de movilidad. Actualmente, la política de emigración de varios países europeos está en trance de conocer evoluciones importantes.

Para posibilitar la gobernanza en esta materia, la Comisión pretende lanzar una gran campaña de sensibilización y de información sobre las posibilidades de movilidad intracomunitaria y sobre el portal de ofertas de empleo EURES. Para ello está haciendo una evaluación sobre un nuevo servicio en línea "Match an Map" (cuyo proyecto piloto fue presentado a finales del 2009) y que va a suministrar información sobre los empleos y la oferta de formación y educación, con el fin de mejorar las capacidades de los trabajadores con los empleos en toda Europa.

La mayoría de los Estados miembros han introducido medidas sociales y de empleo para aliviar el coste humano de la crisis, pero deben ser las políticas europeas las que aporten valor añadido que ayuden a diseñar e implementar respuestas efectivas para luchar contra el paro y a favor de la cohesión social. El impacto social de la crisis está todavía sin calcular, y está siendo más severa de lo esperado, de aquí la importancia de evaluar y mejorar la capacidad de gobernanza europea que permita a través de un enfoque concertado con los agentes sociales reducir el impacto social de la crisis.

\section{LA EVALUACIÓN DEL MODELO SOCIAL EUROPEO ANTE LA CRISIS ECONÓMICA, EN EL CONTEXTO DE LA ESTRATEGIA DE LISBOA}

Los últimos datos, hasta diciembre de 2009, confirmaban que el mercado laboral en la UE continuaba deteriorándose como consecuencia de la crisis económica. El empleo sigue contrayéndose y el desempleo aumentando (cerca de 23 millones), particularmente en hombres y jóvenes. Este deterioro afecta a todos los Estados miembros, pero principalmente a los Países Bálticos, Irlanda y España.

Las recomendaciones políticas específicas para cada país es otro instrumento de gobernanza basado en el Tratado que el Consejo dirige a los Estados miembros sobre 84 la base de una recomendación de la Comisión. En algunos Estados miembros, estas 
recomendaciones han producido un impacto real, estableciendo sus políticas en una dimensión europea. Las políticas de la UE deben de diseñarse y aplicarse a través de una mejor coordinación de los esfuerzos de los Estados miembros, en particular dirigiéndolos a las debilidades estructurales de sus mercados de trabajo, modernizando y mejorando la capacidad de gestión de sus servicios de empleo público y adaptando las políticas a las nuevas realidades y tendencias sociales, sin cambiar los objetivos fundamentales de la Europa Social (las recomendaciones de la estrategia de Lisboa para el crecimiento y el desarrollo).

Esta coordinación debe hacerse, partiendo de la base de que existen en la UE 27 diferentes modelos nacionales de mercado de trabajo y de bienestar Social (ver tipología en Esping-Andersen, 2002; Sakellaropoulos y Berghman, 2004; Davoine, 2008). Una vista general de los mercados de empleo en la UE nos da una imagen muy heterogénea. Se constatan grandes diferencias entre mercados de empleo de Estados miembros, sobre criterios tales como la tasa de empleo, la tasa de actividad y el paro. Estas disparidades reflejan en parte las diferentes instituciones fiscales y de mercado de empleo en los Estados miembros. Utilizando la taxonomía de los autores mencionados con modelos que incluyen los indicadores de Laken (Educación, protección social, condiciones de trabajo, género y reconciliación vida profesional y familiar), junto con otras variables complementarias, podríamos establecer un análisis de grupos. Primero estaría el Nórdico, donde se incluye Dinamarca, Finlandia, Suecia, el Reino Unido y Holanda; luego el Continental donde se incluye Bélgica, Alemania, Austria, Luxemburgo, Francia, Irlanda, Chipre y Eslovenia; en tercer lugar los Países del Sur donde estarían Grecia, Portugal, Italia, Malta y España y por último los nuevos Estados miembros donde se incluyen Polonia, Rumania, Hungría, Bulgaria, Eslovaquia, la Republica Checa, Letonia, Lituania y Estonia.

El valor añadido de la política europea de cohesión social, en esta etapa de crisis económica, debería incidir en aquellos componentes comunes a todos los modelos y que conformarían los contornos del modelo Social Europeo, identificando las prioridades de reformas estructurales en ámbitos tales como educación, formación, salud, protección social y mercados laborales de los Estados miembros de cara a combatir sus debilidades ante la crisis económica. Las discusiones en la UE se han centrado hasta ahora en el impacto de diferentes políticas sobre los resultados de los mercados de trabajo en cada Estado miembro.

En el último informe sobre evaluación ex post del Fondo Social Europeo (14/02/2010) se ha trabajado con esta metodología de análisis de grupos por países, comparando los gastos del FSE con las agregaciones geográficas de países. Se han ido evaluando las medidas del Fondo Social en cada una de las 4 agregaciones geográficas de los Estados miembros establecidas. Lo que ha permitido evaluar la asignación de recursos a nivel de intervención en los Estados miembros, evaluar la política de mercado de trabajo en cada grupo, la adaptabilidad, la inclusión social y el análisis de género. 
La Comisión Europea es consciente de la importancia de las interacciones entre las diferentes políticas de mercado de trabajo en los Estados miembros y las instituciones y cuenta con una herramienta fundamental, como es la evaluación de impacto para mejorar la calidad y la coherencia del proceso de las recomendaciones especificas para cada Estado miembro en su desarrollo de políticas. La evaluación de impacto identifica los posibles efectos positivos y negativos de las políticas propuestas, permitiendo hacer juicios razonados sobre las mismas y señalando los compromisos necesarios para lograr los objetivos perseguidos. La evaluación identifica las buenas prácticas y ayuda a los Estados miembros y los agentes sociales a aprender los unos de los otros. Sin embargo, este instrumento de evaluación no está siendo sólido y transparente y está ayudando poco a una mayor aceptación de las recomendaciones por parte de los Estados miembros.

El proceso de evaluación está destinado a ayudar en la toma de decisiones y no a sustituir el criterio político. El criterio político incluye consideraciones complejas que rebasan con creces la anticipación de las consecuencias de una propuesta. Una evaluación no generará necesariamente conclusiones o recomendaciones tajantes, pero hace una aportación interesante al informar a los responsables de las decisiones sobre las consecuencias de sus opciones políticas. Así, a través de estos análisis de evaluación se está conociendo la eficacia de medidas públicas como respuesta a la deteriorada situación de empleo originada por la crisis. Algunos Estados miembros han introducido o están introduciendo diversas formas de apoyo público, tales como acuerdos temporales sobre reducción de jornadas de trabajo, o la suspensión temporal de la actividad laboral, total o en parte. En estos casos, la pérdida de salario es compensada por el Estado. Estos acuerdos de reducción de tiempo de trabajo a corto plazo están siendo una efectiva forma de preservar el empleo, prevenir el desempleo y mantener el poder de compra a través de compensar las pérdidas de ganancias. Debe quedar claro, que el análisis de la evaluación debe efectuarse a escala europea, buscando el valor añadido de las intervenciones comunitarias comparado con la ausencia de actuaciones por parte de los Estados miembros.

En el año 2005, el relanzamiento de la estrategia de Lisboa, coincidió con la preparación para el periodo 2007-2013 del ciclo de programación de la política de cohesión. Dando una oportunidad a esta política de asignar sus Fondos Estructurales hacia inversiones para el crecimiento y el empleo en los Estados miembros y en concreto para aumentar la eficiencia de sus modelos de empleo y de sus políticas activas de mercado de trabajo.

En un estudio realizado en el 2008 (Good practice in Lisbon Strategy measures por Technopolis Group para la Comisión), se evaluaban las reformas introducidas en la Estrategia de Lisboa, buscando que los recursos de los Fondos Estructurales (la política de Cohesión) se concentrasen en acciones que generasen un mayor impacto en términos de crecimiento y empleo. La "Lisboización " de los Fondos Estructurales ha 86 ayudado a dirigir considerables fondos europeos (unos 228.000 millones de Euros 
durante el período de financiación 2007-2013) a inversiones que fomentan, el crecimiento, como la innovación, la I+D y el apoyo a las empresas. El uso de Fondos Estructurales ha contribuido a que la estrategia de Lisboa sea tangible para las autoridades regionales y locales, que deben desempeñar un papel importante en su aplicación. A través de esta evaluación se ha buscado reforzar la gobernanza de los Fondos Estructurales, considerando posibles sinergias entre los instrumentos de que dispone la UE de cara a maximizar el valor añadido de la Comunidad. Esta evaluación confirmaba que con una mejor coordinación entre los Fondos se mejoraría su gobernanza y la gestión de sus capacidades, a través de diseñar programas óptimos en sectores emergentes, creadores de puestos de trabajo.

La experiencia ha mostrado que el impacto de los Fondos Estructurales puede reforzarse en el futuro, mejorando las estructuras subyacentes (por ejemplo, en investigación e innovación o en los mercados laborales), simplificando los marcos reguladores (por ejemplo, entorno empresarial o desarrollo de infraestructuras) y reforzando la capacidad y la eficiencia administrativa en algunos Estados miembros.

\section{LAS MÚLTIPLES FACETAS DE EVALUAR LA GOBERNANZA EUROPEA EN POLÍTICA LABORAL Y SOCIAL}

La Comunidad Europea apoya los procesos de gobernanza según un enfoque asociativo, a través del diálogo con las autoridades de los países miembros, y como proveedor de fondos con sus diferentes instrumentos financieros.

El concepto de asociación introducido en 2005 ha tenido un impacto positivo en la cooperación y división de responsabilidades entre las instituciones de la Unión Europea y los Estados miembros. El diálogo resultante entre la Comisión y los Estados miembros dio lugar a un intercambio constructivo de impresiones en el que la Comisión aconseja a los Estados miembros sobre las opciones políticas, mientras los Estados miembros ofrecen una perspectiva nacional. El diálogo debe constituir el medio privilegiado para animar a los países a emprender la vía de las reformas. Debe evitarse imponer unilateralmente nuevas condicionalidades. El diálogo ha de tener una dimensión preventiva importante y permitir abordar los aspectos de la gobernanza económica, financiera y social.

En el marco del diálogo deben abordarse las cuestiones de la evaluación de la gobernanza y los progresos realizados, así como las medidas adecuadas ante situaciones de débil capacidad de gobernanza.

Para enmarcar ese diálogo común entre la UE y los Estados miembros están las Directrices Integradas, basadas en el Tratado, que han ayudado a fijar la dirección de las políticas económicas y de empleo nacionales. Aunque las Directrices eran amplias y podrían haber ayudado a sentar la base conceptual de las reformas, su carácter 
genérico y la falta de una jerarquía interna han limitado su impacto en las políticas nacionales. Las Directrices se aprueban por un periodo plurianual y constituyen la referencia fundamental para los programas nacionales de reforma de los Estados miembros (las políticas económicas y de empleo de los Estados miembros), y sólo excepcionalmente admiten modificaciones. La Comisión Europea presentó a finales del 2007 su propuesta sobre las Directrices que fueron aprobadas mediante una Decisión del Consejo de la Unión Europea en julio de ese año. Desde el 2007 se dirigen recomendaciones específicas a los países de la eurozona centradas en las medidas que presentan un interés particular para el buen funcionamiento de la Unión Europea Monetaria (UEM). Estas Directrices están siendo herramientas útiles para promover unas estrategias amplias de crecimiento con mayores vínculos entre las políticas macroeconómica, microeconómica y de empleo.

La aprobación de estas Directrices ha seguido un proceso basado en el Método Abierto de Coordinación (OMC por sus siglas en inglés), otro modo de apoyo de la gobernanza europea, en materia de regulación social. El OMC es un método intergubernamental de "coordinación suave", por el cual los Estados miembros son evaluados por otros Estados miembros y la Comisión juega un papel de vigilancia multilateral proponiendo una regulación dirigida a la convergencia en términos de resultados. Es un método abierto, ya que estimula la cooperación y participación de los diferentes agentes sociales, tratando de promover objetivos e indicadores de resultados comunes para todos los Estados miembros, por medio del consenso. Sin embargo, la mayor parte de los Estados miembros utilizan este instrumento como un informe de prevención y consejo más que de desarrollo político.

A través del OMC se fortalece el sistema de aprendizaje mutuo de los Estados miembros frente a la crisis, al estar orientado no sólo a modificar los resultados de una política, sino también y especialmente el proceso de adopción de decisiones. Este método está centrado en el intercambio de experiencias políticas y en prácticas comunes aprendiendo de ellas a través de la mutua corrección y el consenso (Borras y Jacobson, 2004). Pero este método debe ser reforzado, mejorando la información sobre los resultados y balances.

Es en esta línea que la Comisión Europea anima a los Estados miembros a discutir, en este periodo económico difícil, las nuevas estrategias de cambio y a concebir instrumentos que permitan adecuar mejor las competencias de los trabajadores y desempleados con las ofertas de empleo en sus mercados de trabajo nacionales.

Se facilita de esta manera, una mejor información sobre las necesidades de trabajo en la UE a medio y largo plazo y se completa esta información con proyecciones actualizadas regularmente sobre las tendencias futuras del mercado de trabajo, junto con un análisis de las competencias de los trabajadores por sectores económicos. 88 económica sobre el empleo. 
En el marco del plan de relanzamiento económico propuesto por la Comisión para ayudar a Europa a atenuar los efectos inmediatos de la crisis, nos encontramos, como medida de gobernanza europea, con una amplia iniciativa Europea sobre el empleo, dirigida a promover el empleo y la reinserción en el mercado de trabajo, gracias a medidas de activación, reciclaje y mejora de capacidades de los trabajadores desempleados.

Esta iniciativa ha priorizado dos líneas de actuación concretas en las políticas de empleo de los Estados miembros para 2009 por encima de las Directrices de empleo.

La primera prioridad requiere apostar por un enfoque de actuación integrado que busque facilitar la adaptabilidad de los trabajadores y las empresas y conseguir que la Unión Europea aproveche las ventajas de la globalización. Y aquí es importante resaltar la contribución de los Fondos Estructurales, en particular, el Fondo Social para incrementar la eficiencia de las políticas activas de empleo de los Estados miembros. Evaluar las posibilidades de explotación de este instrumento financiero de la Comisión ha sido uno de los objetivos de la gobernanza que se ha impuesto la Comisión. El acceso al Fondo Social Europeo (FSE) y su utilización han sido simplificados, de tal forma que los pagos del FSE efectuados sobre las cuentas del Tesoro Público de los Estados miembros puedan ser rápidamente entregados a sus beneficiarios.

La segunda prioridad busca la actualización de nuevas aptitudes y capacidades de los trabajadores para explotar plenamente el potencial de relanzamiento económico. Esta prioridad representa un desafío para las autoridades comunitarias, porque el perfil de capacidades de la población varía de un Estado a otro y de una región a otra. La evaluación se presenta como un instrumento esencial para mejorar la preparación y el seguimiento de estas líneas (por ejemplo, la adecuación entre la oferta y la demanda del mercado laboral). La evaluación va a permitir conocer las necesidades emergentes en materia de capacidades a través de un análisis sectorial en la UE, pero también va a permitir a los Estados miembros perseguir la mejora de la calidad y la accesibilidad de la enseñanza y formación.

La mejora de las capacidades para nuevos empleos, independientemente del nivel de cualificación, reviste una importancia capital para el porvenir de Europa. Implica anticipar las capacidades que serán necesarias en los próximos años y adecuar los sistemas educativos y formativos a los nuevos empleos, lo que requiere una colaboración amplia entre instituciones del mercado de trabajo, principalmente servicios públicos de empleo, educativas e interlocutores sociales. Esta tarea es primordial desde el punto de vista de la equidad, dado que los trabajadores poco cualificados son más vulnerables sobre el mercado de trabajo y están sometidos a un mayor riesgo por la crisis. Las políticas de educación, de la formación y del empleo de los Estados miembros deben de concentrarse sobre el reforzamiento y adaptación de competencias y la mejora de la formación a todos los niveles, con el fin de conseguir en la Unión una mano de obra altamente cualificada y sensible a las necesidades de la economía. 
Para mejorar la capacidad de gobernanza en materia social, la UE debería animar a los Estados miembros a reformar sus sistemas de educación y formación de acuerdo con los desafíos que plantea la globalización y la transición hacía una economía del conocimiento, y los cambios que se aprecian en las tendencias demográficas. Todo ello realizado en un marco donde debe estar presente la participación, el intercambio de información y conocimiento, y la transparencia, la efectividad y la eficiencia, así como el seguimiento de las reglas del Derecho Comunitario.

Para poder hacer un análisis de calidad de la gobernanza social europea, los Estados miembros presentan informes de progreso basados en indicadores económicos y sociales adecuados, que permiten valorar los avances alcanzados durante el año y conocer los resultados de las actuaciones para contrarrestar los efectos de la crisis.

Ello exige un diálogo regular y transparente de la UE con los Estados miembros con el fin de apreciar las tendencias y evoluciones de estos indicadores. Es importante animar a los gobiernos de los Estados miembros, agentes políticos, organismos públicos independientes a que desarrollen sus propias herramientas y capacidades para definir las reformas necesarias, que permitan calibrar la situación y los progresos realizados.

\section{LA EVALUACIÓN DE LAS INSTITUCIONES DEL MERCADO LABORAL EN TIEMPOS DE CRISIS EN LA UE COMO SOPORTE DE GOBERNANZA}

En el titulo VII del Tratado constitutivo de la Comunidad Europea se establecen los principios y procedimientos para desarrollar una estrategia coordinada de empleo en el ámbito europeo.

En 1997 La Estrategia Europea de Empleo (SEE) fue lanzada para coordinar los esfuerzos y las acciones de los Estados miembros, así como para implementar sus políticas. Desde el año 2000, y dentro del marco de la Estrategia de Lisboa, la SEE ha sido enriquecida con elementos importantes dentro de la política europea de empleo. Doce años más tarde del lanzamiento de esta estrategia, la evaluación de la misma recomienda qué Comisión debería fortalecer las recomendaciones dirigidas a los Estados miembros y promover reformas en sus sistemas de empleo.

Uno de los objetivos claves ante la crisis es mejorar sustancialmente las Instituciones de los Estados miembros y de la Unión para prever, anticipar y adecuar entre sí las futuras capacidades y exigencias del Mercado Laboral. Esto es necesario para ayudar a los trabajadores desempleados a reintegrarse al mercado de trabajo, y para orientarles en el desarrollo de sus competencias, mejorando las perspectivas de empleo a largo plazo. Las Instituciones del mercado de trabajo y en particular los Servi90 de la crisis en el empleo. 
Eliminar obstáculos para lograr la libre circulación de trabajadores en la UE, mejorar la transparencia de información sobre las tendencias del mercado de trabajo y sobre los planes de evaluación y de adecuación de las competencias de los trabajadores, prevenir riesgos de paro estructural constituyen funciones fundamentales a desempeñar por estos organismos si se quiere conseguir una elaboración de políticas eficaces en materia de empleo.

La estrategia de la Comisión de cara a la crisis ha estado en todo momento dirigida a ayudar a los Estados miembros a concebir y a poner en práctica políticas de empleo adaptadas y eficaces, definiendo prioridades tales como: preservar los empleos existentes; creando nuevos y estimulando la movilidad; desarrollar las capacidades y responder a las necesidades del mercado de trabajo; mejorar el acceso al empleo.

Lograr esto supone incrementar las capacidades metodológicas, analíticas y de aprendizaje mutuo entre las instituciones laborales en la UE, para la previsión de las necesidades de capacitación y de puestos de trabajo. Estas instituciones tienen que establecer líneas de actuación y servicios que puedan atender a las necesidades de los desempleados y a las inadecuaciones del mercado laboral. Los esfuerzos de modernización de SPE en los últimos años, está posibilitando el que exista una mejor transparencia del mercado laboral en la UE.

Bien es cierto que las previsiones que hagan las instituciones no puede suministrar una imagen precisa del futuro, pero ellas puede dar una indicación de las tendencias generales, a través de facilitar una mejor información sobre las necesidades de capacitación a medio y largo plazo en la UE. Por eso, conocer cómo y con qué instrumentos deberían las instituciones laborales enfrentarse a los desafíos, presentes y futuros del mercado laboral, constituye actualmente en la UE un tema de preocupación y de evaluación. La actualización periódica de las previsiones sobre las tendencias futuras en los planos macroeconómico, demográfico y del mercado laboral a niveles local, regional, nacional y europeo, reviste una importancia particular en la óptica de adaptación al cambio, que corresponde atender a estas Instituciones. La Comisión Europea está llevando a cabo en este periodo (2007-2013) evaluaciones sobre las reformas de los Servicios Públicos de Empleo; destacamos como primera experiencia la evaluación llevada a cabo en Polonia para valorar los cambios habidos en la Administración de este país, dentro del proceso de modernización y analizar su repercusión en las intervenciones del FSE y en su política de empleo. Los resultados de esta evaluación han revelado las deficiencias existentes en la programación e implementación en las intervenciones del FSE. No existía una clara lógica de conexión entre los niveles de proyecto, la justificación y los objetivos de acciones individuales, así como entre el objetivo de la actividad y la prioridad de los ejes. En varios proyectos se encontró que no había conexión entre los resultados y los problemas a resolver en los mismos. La Comisión analiza las posibles soluciones a estos problemas y propondrá instrumentos y métodos para resolverlos.

Bien es cierto que no existe un paradigma lógico que garantice la Buena Gobernanza. No es posible indicar qué modelo de gestión pública debe seguir un programa 
de actuación del FSE para lograr sus resultados. Sin embargo, es importante tener un instrumento de aprendizaje sobre el cual poder reflejar, adaptar y cambiar, si es necesario las políticas.

El concepto de capacidad Institucional y Administrativa en la UE y en los Estados miembros subraya la atención que hay que dar al desarrollo de estrategias y competencias para maximizar las oportunidades de la política del mercado laboral. Aspectos típicos de capacidad son la calidad de la formación de los funcionarios, las características de organización del trabajo, las relaciones intergubernamentales y el estilo de interacción entre el gobierno y su medio social.

El desarrollo del capital humano y social de las Administraciones públicas es un soporte esencial para realizar las reformas estructurales que requiere la crisis económica. Unas efectivas estructuras institucionales son una importante fuente de innovación y competitividad. Por ejemplo, en Portugal, la reforma de los servicios públicos ha permitido que el número de días para crear una empresa haya pasado de 60 a 12. Lo mismo ocurre con la legislación comunitaria, muchas áreas de la renovada estrategia de Lisboa deben ser mejor diseñadas políticamente, para que se puedan darse las condiciones adecuadas de crecimiento económico y creación de empleo.

Cualquier acción conjunta dirigida a mejorar la capacidad administrativa e institucional de los Estados miembros y de las regiones europeas podrá contribuir a alcanzar los objetivos de la renovada estrategia de Lisboa en materia laboral, asegurando una buena gobernanza europea, gracias a un diseño, implementación y evaluación de sus políticas y programas de manera participativa, coordinada y transparente.

El desarrollo e implementación de políticas efectivas en esta materia requiere la participación y cooperación de los actores sociales para identificar las principales debilidades de las Administraciones y para que puedan llevarse a cabo las necesarias reformas en materia laboral. Esta disponibilidad de los actores sociales constituye un factor determinante para los Estados miembros para poder aprovecharse de todos los beneficios de la gobernanza de la Unión y a su vez una condición necesaria para lograr encontrar soluciones de salida a la actual crisis económica.

\section{LA EVALUACIÓN DEL FSE COMO INSTRUMENTO FINANCIERO QUE SOSTIENE LA GOBERNANZA DEL MODELO SOCIAL EUROPEO}

El Fondo Social Europeo (FSE) es un instrumento financiero que representa una décima parte del presupuesto comunitario, a través de él se benefician 9 millones de personas cada año en la UE. Contribuye a preservar los valores del modelo social europeo, respaldando el empleo y combatiendo el desempleo, también cofinancian actividades que favorecen el desarrollo y fortalecimiento de capacidades 92 administrativas tradicionalmente centradas en servicios de empleo y educación. 
Durante estos últimos diez años el FSE, ha permitido poner en práctica la estrategia europea para el empleo y ha constituido un medio esencial para la realización de los objetivos de Lisboa. Así, más del 90\% de los 76.000 millones de euros que representa el presupuesto del FSE para el periodo 2007-2013, está concentrado en las prioridades de la estrategia de Lisboa.

En este periodo 2007-2013, los Fondos Estructurales tienen sus recursos concentrados en tres objetivos:

a) Convergencia, dirigido a ayudar a las regiones menos desarrolladas de los Estados miembros, mejorando sus condiciones de crecimiento y empleo.

b) Competitividad dirigido a fortalecer la competitividad y el empleo de todas las regiones europeas.

c) Cooperación territorial dirigida al fortalecimiento de proyectos transnacionales e interregionales a nivel europeo.

La regulación del Fondo Social pone especial énfasis, actualmente en la promoción de la gobernanza a través del desarrollo de conexiones y redes de varios interlocutores sociales europeos, con efecto positivo en la formación para mantener a las personas en el empleo y ayudarlas a encontrar nuevos puestos de trabajo. En general el impulso de las actividades del FSE se centra en fomentar el cambio y mitigar los efectos de la crisis económica en el empleo. Así pues, a medida que la crisis económica se hace extensiva a la economía real, las prioridades de financiación del FSE cobran más importancia. Por ejemplo el FSE contribuye a:

- Descubrir y aplicar formas de trabajar más innovadoras y productivas.

- Acelerar el apoyo a los trabajadores y a las empresas para adquirir las nuevas aptitudes que necesitan cuando la economía vuelva a crecer con normalidad.

- Aplicar medidas activas y preventivas para fomentar empleo. La crisis económica puede acelerar cambios, por ejemplo a través de iniciativas de formación que permitan a los trabajadores reciclarse hacia otros sectores donde la demanda sea mayor.

- Respaldar el autoempleo y la creación de empresas. Los fondos del FSE se utilizan para ayudar a los empresarios y desempleados a crear sus propias empresas, proporcionándoles orientación y apoyo.

EI FSE persigue los principios de coherencia y concentración. Coherencia con los objetivos y prioridades de la estrategia de Lisboa y Concentración de recursos en aquellas áreas geográficas donde tengan mayores necesidades de empleo. A lo largo del año 2009, la Comisión lanzó una evaluación sobre la contribución del FSE a la Es- 
trategia Europea por el Empleo (EEE). El objetivo era conocer el valor añadido de las intervenciones del FSE en los Estados miembros y como se han logrado los objetivos de la EEE. El trabajo debería vincular la identificación de las intervenciones lógicas del FSE con los impactos y los efectos que persigue las distintas directrices de la EEE.

El Fondo Social Europeo constituye una herramienta clave para apoyar la recuperación económica en Europa, junto con otros instrumentos y políticas de la UE que integran el paquete de esfuerzos concertados para recuperar el crecimiento y generar empleo [Fondo Europeo de Desarrollo Regional (FEDER), Fondo Europeo de Adaptación a la Globalización (FEAG), Banco Europeo de Inversiones)].

A lo largo de 2009, se han presentado por parte de la Comisión propuestas de simplificación de criterios para el apoyo del FSE al Plan de Recuperación con adelantos de pagos a principios de 2009 y la revisión de las normas del FEAG.

EI FEAG es un instrumento de acción rápida como parte de la respuesta europea a la crisis económica y financiera. Ayuda a trabajadores en procesos de reestructuración, afectados por la crisis y no sólo por el impacto de la globalización.

La Comisión Europea, siguiendo los criterios de gobernanza, debate con los representantes de los interlocutores sociales las propuestas de acciones que van a ser financiadas por el FSE destinadas a aumentar el crecimiento y el empleo, e intenta consolidar y reestructurar el mercado laboral con el fin de prepararlo de cara al futuro.

Es importante que estos representantes consideren la gobernanza con la Comisión como una prioridad, compartiendo con ella los instrumentos de análisis y evitando emprender diálogos individuales que apoyen reformas del mercado laboral de manera unilateral y no coordinada. Aunque los instrumentos de acción disponible para atacar el paro y estimular la demanda está en manos de los Estados miembros, la acción comunitaria tiene que aportar un valor añadido a las medidas nacionales. La UE debe adoptar y financiar a través de sus instrumentos financieros, medidas a corto y medio plazo que permitan salir de la crisis.

Medidas como aumentar inversiones en I+D+l.; mantener los niveles de empleo mediante la flexiseguridad y la movilidad, y reforzar la competitividad europea, haciendo nuestra economía más ecológica, son las que van a permitir crear nuevos empleos y desarrollar nuevas tecnologías. Pero todo ello debe hacerse basándose en una gobernanza de paternariado, donde los Estados miembros se pongan de acuerdo sobre los niveles de coordinación más elevados para hacer frente a estos desafíos. Por ejemplo, en el desarrollo de la flexiseguridad es importante asumir un enfoque que abarque diferentes tipos de flexibilidad del mercado de trabajo, y que contemple medidas de seguridad laboral para cada uno de estos tipos de flexibilidad. En otras 94 palabras, saber diseñar mecanismos de flexibilidad que permitan la tra 
El apoyo financiero a estas medidas por parte del FSE debe hacerse también en base a principios de gobernanza tales como: Apertura (las Instituciones deben trabajar de forma abierta). Participación (la participación de las Instituciones depende de un enfoque integrador que genere una mayor confianza en los resultados). Responsabilidad (que se clarifique el papel de cada uno de los que intervienen en el proceso). Eficacia (que se produzcan los resultados buscados sobre la base de unos objetivos claros). Por último, Coherencia (requiere un liderazgo político y un firme compromiso de las instituciones); promoviendo programas de gastos más innovadores, flexibles y de mayor valor añadido. Sólo de esta forma se puede ayudar a la sostenibilidad del Modelo Social Europeo y a que pueda hacer frente a la nueva estrategia de cambio que requiere la crisis económica. En esta línea, la Comisión está proponiendo a los Estados miembros una modificación del Reglamento sobre los Fondos Estructurales, para financiar medidas de relanzamiento rápido, acelerando la realización de proyectos dirigidos a: ayudar a trabajadores o empresas enfrentados a una reestructuración, o la adaptación de competencias de los trabajadores a las necesidades del mercado, y a los jóvenes, y grupos más vulnerables a través de becas o préstamos en el campo de la formación y de la educación formal.

\section{EVALUACIÓN Y GOBERNANZA EN EL MARCO DE LA PROPUESTA DE LA COMISIÓN PARA COMBATIR EL IMPACTO SOCIAL DE LA CRISIS}

La Comisión Europea ha propuesto a lo largo del 2009 una serie de iniciativas, desde el Plan de Recuperación, hasta los cambios en el FSE pasando por iniciativas como mejora de las cualificaciones y adecuación entre empleo y necesidades, así como la publicación de informes mensuales de observación de los mercados de trabajo y de comunicaciones para ayudar a los Estados miembros a diseñar e implementar apropiadas y efectivas políticas de empleo como respuesta a la crisis. Todas ellas fueron tenidas en cuenta en el Consejo Europeo de Primavera de ese año y en la cumbre para el empleo de mayo 2009. Dichas iniciativas fueron dialogadas con los Estados miembros y con los Agentes Sociales para definir las tres prioridades claves en que se tendría que centrar la política de empleo comunitaria:

- Mantenimiento y creación de empleos.

- Actualización de competencias y ajuste a las necesidades del mercado de trabajo.

- Incrementar el acceso al trabajo.

Todas las iniciativas han sido coherentes y consistentes con la estrategia de Lisboa y la Estrategia Europea para el empleo. Utilizando esta última como marco adecuado para coordinar las acciones y promover el empleo en tiempo de crisis. 
La coyuntura actual está haciendo que la Comisión establezca ciertas prioridades en las políticas de empleo de los Estados miembros por encima de las Directrices que marca el Modelo Social Europeo. Estas prioridades de actuación que requieren una acción inmediata está exigiendo la adaptación y adecuación de las medidas contenidas en la Estrategia de Lisboa a la crisis actual, en otras palabras reformar el Modelo Social Europeo.

La reforma del Modelo Social Europeo es uno de los debates más complejos sobre los que se enfrenta la UE. Este modelo es el resultado de un largo proceso histórico intentando combinar la justicia social con el desarrollo económico. La crisis actual está haciendo que la estrategia renovada de Lisboa (2008-2010) para la que se habían definido las nuevas directrices de Política Económica y Empleo a finales de 2007, se enfrente a unos avances muy limitados y que tenga que replantearse alcanzar los objetivos previstos para 2010. Aunque esto no debía significar renunciar a la Estrategia de Lisboa como referencia a medio plazo, sin embargo habrá que reconsiderar su reformulación una vez superada la crisis.

Hoy en día, está claro que el Modelo Social Europeo tiene que hacer frente a nuevos desafíos estratégicos (Rodrigues, 2006) como pueden ser:

- La globalización y las nuevas presiones competitivas.

- La transición hacia una economía intensiva del conocimiento.

- Las tendencias demográficas.

- Los nuevos modelos familiares.

- El nuevo proceso de integración Europea, en su nueva etapa, adoptando un marco común de nuevas competencias.

La sostenibilidad del Modelo Social Europeo depende de cómo se reformen sus componentes y se adapten a estos desafíos. La crisis es en sí misma un gran desafío sobre el potencial crecimiento de la Unión, la creación de empleos, y sobre el desarrollo sostenible de los standards de vida. El acceso a nuevas competencias será crucial para obtener nuevos y mejores trabajos. Así, la educación y la formación continua deberán ser reformadas para hacer frente a los desafíos de la globalización y la transición a la economía del conocimiento.

Las limitaciones del gasto público en los Estados miembros tendrán un gran impacto sobre las políticas de empleo de los próximos años; los sistemas de protección social necesitarán incrementar su eficiencia y adecuarse para mantener su sostenibilidad. De aquí la importancia de los procesos de evaluación externos, para conocer el impacto de las reformas y el funcionamiento del proceso de coordinación entre las 96 políticas de los Estados miembros. Sintetizar y comparar los resultados de medidas 
sobre políticas de empleo. Identificar los factores de eficiencia que han contribuido a esas políticas. Elaborar conclusiones y formular recomendaciones para cada medida dirigida al empleo, o a la formación continua, son tareas imprescindibles para poder satisfacer las necesidades del modelo de bienestar social que los Estados miembros quieren promover.

Si tenemos en cuenta la tipología de modelos de bienestar en Europa, mencionada anteriormente, las reformas tendrían que tener en cuenta las peculiaridades de cada uno:

- En el modelo continental las medidas estarían dirigidas a equilibrar los beneficios sociales entre los empleos temporales y permanentes o a promover un modelo sostenible de protección social.

- En el modelo anglosajón el énfasis se pondría en construir un modelo inclusivo de protección social y en desarrollar servicios de guardería.

- En el modelo de los países del sur se pondría el énfasis en hacer frente simultáneamente a los desafíos de baja seguridad en el empleo y baja flexibilidad.

- Mientras que en el modelo escandinavo se buscarían nuevas soluciones para la flexiseguridad.

La evaluación de estas medidas deberá ir dirigida a evitar una polarización de la expansión de empleo en los mercados de trabajo de los Estados miembros, con un aumento de desigualdades entre los mismos, incluso entre las ocupaciones con mayores y menores cualificaciones. El informe de la Fundación Europea sobre condiciones de vida y trabajo de febrero del 2008, señala que los nuevos trabajos creados entre 2000 y 2006, aunque han mejorado en calidad, sin embargo están creando distintos modelos de expansión de empleo, con una polarización perceptible entre las cualificaciones demandadas en algunos Estados miembros.

\section{CONCLUSIONES}

\section{- La necesidad de evaluación continua de la gobernanza europea en materia laboral}

Después del 2010, la principal responsabilidad para desarrollar los mercados de trabajo y las políticas sociales quedarán no sólo para los Estados miembros, sino también para las instituciones de la UE. La diversidad de situaciones entre y dentro de los Estados miembros requerirán diferentes enfoques, y aquí aparece la oportunidad para las Instituciones Europeas de demostrar cuál es su valor añadido. La credibilidad de Lisboa y de la Estrategia Europea para el empleo en estos tiempos de crisis, dependerá de las reformas, innovaciones y efectividad de las políticas de empleo. Las prio- 
ridades políticas que hemos descrito en los anteriores apartados van a seguir siendo pertinentes en el futuro de la Estrategia Europea de Empleo.

La nueva gobernanza europea en materia laboral estará dirigida a mejorar la coordinación de políticas entrelazadas, centrarse en prioridades objetivo y simplificar los sistemas de gestión, mejorar la participación y la supervisión entre pares (peer review). En la iniciativa de la Comisión "Competencias nuevas para empleos nuevos" (Comunicación de la Comisión, 2009) se señala la necesidad de aumentar la eficacia de las políticas de educación y de formación y de modernizar los mercados de trabajo gracias a políticas de flexiseguridad. La Comisión en paternariado con los Estados miembros tendrá que evaluar la eficacia de medidas dirigidas hacia estos objetivos de manera continua en los próximos años. La elaboración de las políticas de mercado de trabajo y políticas sociales seguirá siendo competencia de los Estados miembros, pero la diversidad de las situaciones en cada Estado miembro obligará a un tratamiento por parte de la Comisión más diferenciado y participativo.

La importancia de la evaluación ante las nuevas iniciativas comunitarias aparece para examinar en qué contexto y con qué mecanismos deben ser aplicadas las políticas de empleo y cuáles son los factores de eficiencia que deben ser utilizados por los Estados miembros (tipos de estrategia, tipos de estructuras y sistemas en que se apoyan, tipos de beneficiarios de medidas, acuerdos de cofinanciación, redes y colaboraciones, concentración de las ayudas).

A través de la evaluación de estas políticas de empleo y de sus redes de actores podemos identificar cuales de ellas pueden ser desarrolladas a nivel europeo y a nivel nacional, para poder tener una visión general de las reformas de los mercados laborales e identificar los puntos críticos de estas reformas de acuerdo con los diferentes modelos de bienestar social existentes en la Europa Comunitaria.

\section{- El nuevo contexto de evaluación de la estrategia "Europa 2020"}

La estrategia “Europa 2020" y la revisión de la Estrategia Europea de Empleo forman parte del contexto de gobernanza europea futura, con unas nuevas perspectivas en materia de capacidades (Nuevas cualificaciones para nuevos empleos), identificación de políticas innovativas (Plan de innovación UE), nuevos vínculos de integración entre empleo (Nueva agenda de empleo) e inclusión y protección social (Acción europea contra la pobreza), y nuevos mecanismos de responsabilidad y vigilancia multilateral. Todos estos proyectos que forman parte de la gobernanza europea, tienen que ser evaluados dentro de un contexto que permita coordinar mejor las políticas sociales y de empleo en la UE. Las Directrices Europeas de empleo que surjan después del 2010, deberán ser simplificadas, con responsabilidades más claras entre el EM y la Comisión, más centradas en los resultados que en la gestión y articularse alrededor de objetivos más precisos con el fin de garantizar una mayor coherencia. 
ropa 2020 debe de estar dotada de una gobernanza fuerte que permita la puesta en práctica de las tres prioridades para un crecimiento y un empleo sostenible como son: Crecimiento basado en el conocimiento y la innovación. Una sociedad inclusiva con altos niveles de empleo. Crecimiento verde: una economía competitiva y sostenible. Europa 2020 debe ser el resultado de una evaluación seria sobre lo que pretende ser la política económica europea, si se quiere sentar las bases de una verdadera gobernanza económica para Europa.

Los Estados miembros deberán elaborar políticas dirigidas a estas prioridades que estimulen el crecimiento y el empleo, aumenten los niveles de competencia y sepan explotar el papel positivo de las políticas de inclusión en materia de empleo y viabilidad financiera. Para ello es muy importante que sea reforzado el diálogo social en la elaboración y reformas del mercado laboral y que se destaque el papel de coordinación de la Comisión y los Estados miembros con los agentes sociales para facilitar las comparaciones transnacionales y el aprendizaje mutuo.

\section{REFERENCIAS BIBLIOGRAFÍCAS Y DOCUMENTALES}

Barca, F. (2009), An Agenda for a Reformed Cohesion Policy. Informe independiente. Bruselas: Commissioner for Regional Policy.

Borrás, S. y Jacobsson, K. (2004), “The Open Method of Coordination and new governance patterns in the EU", Journal of European Public Policy, 11(2): 185-208.

Consejo Europeo (2008), Conclusiones de la Presidencia 11 y 12 de diciembre de 2008 y Comisión Europea. Un Plan Europeo de Recuperación Económica. Comunicación de la Comisión de 26 de noviembre. Bruselas: Consejo Europeo.

European Foundation for Living and Working Conditions (2008), “More and better Jobs: Patterns of employment expansion in Europe". Foundation Paper, 1, febrero. Luxemburgo.

Daly, M. (2006), “EU social policy after Lisbon”, Journal of Common Market Studies, 44(3): 461-481.

Davoine L., Erhel C., Guergoat-Larivière M. (2008), “A taxonomy of European labour market using quality indicators". Working Paper halshs-00317280_v1, Université Paris 1. Pantheon-Sorborn.

Esping-Andersen, G. (1990), The three worlds welfare capitalismo. Cambridge: Policy Press.

European Commission (2001), Overview of the final evaluations of the ESF co-funded programmes (Note on the evaluation of the contribution of the structural funds to the European Employment Strategy 2001). Bruselas: European Commission.

European Commission (2005), Evaluation on the ESF Contribution to Employment, Inclusion and Education \& Trainig policies though. The support to Systems and Structures 2005. Bruselas: European Commission.

European Commission (2007), Evaluation of the Integrated Guideline Package for Growth and Jobs and the Related Cooordination. Bruselas: Euréval y Ramboll Management. 
European Commission (2008), Employment in Europe 2008. Luxemburgo: Dirección General de Empleo, Asuntos Sociales e Igualdad de Oportunidades.

European Commission (2008), Comunicación sobre New Skills for New Jobs Anticipating and matching labour market and skills needs. Bruselas: Commission of the European Comunities, COM: 868.

European Commission (2008), Evaluation of contribution of the ESF to the European Employment Strategy 2010. Bruselas: Euréval y Ramboll Management.

European Commission (2009), Monthly monitor on the EU employment situation and social outlook. European Commission Communication for the Spring European Conuncil, Driving European Recovery. Bruselas: Commission of the European Comunities, COM: 114 .

European Commission (2009), Ex post Evaluation of the European Social Fund. Informe elaborado por Consortium LSE Enterprice, Vision \& Value, Red2Red, referencia del contrato: VC/2008/0693.

European Commission Lisbon (2010), "Strategy evaluation”, Document SEC: 114-final. Lonnroth, J. (2000), "The European Employment Strategy, a model of open coordination and the role of social Partners". Conferencia presentada en SALTSA/Swedish National Institute for Working Life. Bruselas, 9-10 octubre.

Parlamento Europeo (2009), Resolución del Parlamento Europeo de 5 de marzo de 2009, sobre la contribución al Consejo Europeo de primavera de 2009 en relación con la Estrategia de Lisboa, (11 de marzo de 2009) Estrasburgo. Procedimiento: 2008/2644(RSP).

Président de la Commission Européenne (2010), EUROPA 2020: Stratégie pour une croissance et des emplois durables. Contribution du président de la Commission européenne en vue de la réunion informelle des chefs d'Etat ou de gouvernement du 11 février 2010. Bruselas: Commission of the European Comunities, COM.

Rodrigues, M.J. (2006), The new knowledge Economy in Europe-a strategie for International competitiveness and social cohesion. Cheltenham: Edward Elgar.

Rodrigues, M. J. (2006), Flexicurity References and tools for policy choices, Bruselas. En línea: <http://ec.europa.eu/employment_social/employment_strategy/index_fr.htm.Juin2006> (Consulta: marzo 2010).

Recibido: 18 de febrero de 2010

Aceptado: 7 de abril de 2010 